\title{
Dynamics in supramolecular polymer networks formed by associating telechelic chains
}

Article

Accepted Version

Amin, D., Likhtman, A. E. and Wang, Z. (2016) Dynamics in supramolecular polymer networks formed by associating telechelic chains. Macromolecules, 49 (19). pp. 7510-7524. ISSN 0024-9297 doi:

https://doi.org/10.1021/acs.macromol.6b00561 Available at https://centaur.reading.ac.uk/66960/

It is advisable to refer to the publisher's version if you intend to cite from the work. See Guidance on citing.

To link to this article DOI: http://dx.doi.org/10.1021/acs.macromol.6b00561

Publisher: American Chemical Society

All outputs in CentAUR are protected by Intellectual Property Rights law, including copyright law. Copyright and IPR is retained by the creators or other copyright holders. Terms and conditions for use of this material are defined in the End User Agreement.

www.reading.ac.uk/centaur 
Central Archive at the University of Reading

Reading's research outputs online 


\title{
Dynamics in Supramolecular Polymer Networks Formed by Associating Telechelic Chains
}

\author{
Dipesh Amin, Alexei E. Likhtman and Zuowei Wang*
}

September 22, 2016

School of Mathematical, Physical and Computational Sciences, University of Reading, Whiteknights, Reading RG6 6AX, UK

\begin{abstract}
We present hybrid molecular dynamics/Monte Carlo simulations of supramolecular networks formed by unentangled telechelic chains with sticky end monomers (or stickers) of finite functionality. The reversible bonding between sticky monomers leads to the formation of sticker clusters with well-defined size distribution, which in turn work as cross-links for transient polymer networks. We study the kinetics of sticky monomer association, the topological structure and the resulting dynamic and rheological behavior of the supramolecular systems as a function of the sticker bonding energy $\varepsilon$ and the parent polymer chain length. Percolated transient networks are formed above a threshold bonding energy around $4.3 k_{B} T$. At high bonding energies $\varepsilon \geq 10 k_{B} T$, the majority of the stickers are fully reacted and the fraction of open stickers is less than $1 \%$. The conventional picture of a single sticker hopping from one cluster to another is energetically unfavorable. We find the dynamic and rheological behavior of such strongly associated supramolecular networks are dominated by a partner exchange mechanism in which the stickers exchange their associated partners, and so release the imposed topological constraints, through the association and disassociation of sticker clusters. The characteristic time of the partner exchange events grows exponentially with the bonding energy and is up to 2 orders of magnitude longer than the average lifetime of the reversible bonds. As
\end{abstract}


a result, three relaxation regimes can be clearly identified in the stress and chain end-to-end vector relaxation functions as well as the mean-squared displacements of the stickers, which are the initial Rouse regime, the intermediate rubbery regime and the terminal relaxation regime. A phantom chain hopping model based on the microscopic understanding is proposed to describe the chain relaxation dynamics in the supramolecular networks, which provides numerical predictions in reasonably good agreement with our simulation results. 


\section{Introduction}

Supramolecular polymer networks are formed by physical association of linear or branched polymers via reversible non-covalent bonds, ${ }^{1-4}$ such as hydrogen bonds, ${ }^{5-10} \pi-\pi$ stacking, ${ }^{11,12}$ metal-ligand ${ }^{13-15}$ and ionic interactions. ${ }^{16-19}$ The reversibility of crosslinking provides them unique abilities for working as self-healing, stimuli-sensitive and shapememory materials. They also have superior processing and recycling properties over traditional polymers and chemical networks constructed from covalently crosslinked polymers owing to the sharp decrease in viscosity upon increasing temperature or decreasing concentration. The potential applications of supramolecular polymer networks have inspired strong interests in understanding the physical mechanisms underlying their structural, dynamic and mechanical properties. ${ }^{1-4}$

The topological structures of supramolecular polymer networks are determined by the molecular composition of the parent polymers and the nature of the non-covalent interactions. The associating polymers which form supramolecular polymer networks can be classified into two main groups according to the chemical distribution of associating groups or stickers. In one group, the associating polymers have multiple stickers distributed along their backbones. Each polymer can thus be cross-linked with several other polymers at well-separated bonding sites. ${ }^{10,16,20,21}$ The simplest and most widely studied supramolecular systems in this category are those formed by the pairwise association of stickers. ${ }^{17,21,22}$ As the sticker functionality increases above two, they begin to aggregate into clusters which effectively work as cross-links in the transient network. Since each sticker is chemically connected to two chain segments, these clusters are usually relatively small due to steric hindrance and chain stretching. In the other group the associating polymers have the stickers only at their extremities, such as telechelic and triblock polymers. ${ }^{23-25}$ A sticker functionality no less than three is required for linear polymers in this category to associate into three-dimensional (3D) networks. Supramolecular networks formed by these polymers typically consist of large aggregates of stickers or flower-like micelles bridged by flexible polymer chains. The complicated topological structures, to- 
gether with the interplay between the dynamics of the parent polymer chains and the breaking/reforming kinetics of the physical bonds, leads to the rich dynamic behavior of supramolecular polymer networks. The intrinsic characteristic times include, but are not limited to, the lifetime of the reversible sticky bonds $\tau_{b}$, the entanglement time $\tau_{e}$, the Rouse time $\tau_{R}$ and the reptation or terminal time $\tau_{d}$ of the parent polymers. The system behaves like a permanent polymer network at time scales smaller than the bond lifetime, and as a standard polymer melt or solution when the bonding constraints are fully released. The most fascinating properties of supramolecular polymer networks are associated with the relaxation dynamics in between these two time limits.

Theoretical models have been developed for describing the dynamic and rheological properties of supramolecular polymer networks in accordance with their structural classification. ${ }^{21,22,26-33}$ For transient networks formed by unentangled polymers with many pairwise associating stickers per chain, the sticky Rouse model developed by Rubinstein and coworkers predicts that the Rouse relaxation time of the chains is proportional to the renormalized bond lifetime $\tau_{b}^{*}\left(\gg \tau_{b}\right)$ times the square of the number of interchain sticky bonds per polymer. ${ }^{22}$ The renormalization arises from the fact that a sticker needs to experience many breaking and reforming events with its old partner before finding another open sticker to associate with. The sticky reptation model extends this idea to entangled polymers with pairwise associating stickers by considering that the polymer performs sticky Rouse motion along the contour of the confining tube. ${ }^{22}$ The sticky reptation time is thus given by the sticky Rouse time multiplied by the number of entanglements per chain. A scaling theory based on the assumptions of pairwise association and hopping diffusion of stickers has also been developed to describe the self-healing process of unentangled supramolecular polymer networks. ${ }^{34}$

If the stickers are able to aggregate into large clusters, leading to reversible networks of interconnected micelles, two mechanisms, namely polymer chain diffusion ${ }^{27,31}$ and positional rearrangement of the micelles, ${ }^{31}$ have been proposed to relieve stress collectively. In these systems, the hopping of stickers is assumed to proceed by dissociating from one micellar core and then associating into another. Marrucci et al. predicted a 
power-law dependence for the terminal relaxation time of unentangled telechelic chains on the polymer concentration and molecular weight. ${ }^{27}$ For associating polymers with many regularly spaced stickers, Semenov and Rubinstein predicted that the chain relaxation time has a power-law dependence on polymer concentration in the unentangled or weakly entangled regime, but an exponential concentration dependence in the strongly entangled regime. ${ }^{31}$ Unlike the pairwise association case, ${ }^{22}$ the bond lifetime renormalization is considered negligible when the sticker dissociation energy is in the range of $M^{1 / 2}<\varepsilon / k_{B} T<M^{4 / 3}$ with $M$ the average aggregation number of sticker clusters. ${ }^{31}$ This is because the aggregates can accommodate a varying number of stickers and the estimated energy change before and after a sticker hopping event is lower than the thermal energy $k_{B} T$. The terminal stress relaxation time of these networks is determined by the micellar positional rearrangements, which is exponentially longer than the single-chain relaxation time due to high energy barriers. ${ }^{31}$

A number of experimental works have been carried out to test the predictions of the above-mentioned theoretical models and qualitative agreements have been found on the diffusion and rheological behavior of certain associating polymer networks. ${ }^{17,27,35,36}$ For example, Colby and coworkers have shown that the sticky Rouse model can well describe the linear viscoelasticity of polyester ionomers when using the ionic association lifetime measured in dielectric relaxation spectroscopic responses as model input parameters. ${ }^{17}$ But there is still a lack of microscopic evidence to validate the assumptions made in the theoretical models, such as the microscopic description of the sticker hopping process and positional rearrangement of micelles. Computer simulations at the atomistic or finegrained level can help to provide such microscopic insights which are generally difficult to access in experiments.

Simulation studies on associating polymers have been mostly focused on static properties, in particular the sol-gel transition and the aggregation of associating groups. ${ }^{37-46}$ Much less attention has been paid to the dynamic and rheological properties and their relation to the topological structures and parent chain dynamics. ${ }^{47-50}$ Hoy and Fredrickson applied hybrid molecular dynamics/Monte Carlo (MD/MC) simulations to study 
supramolecular networks formed by unentangled associating polymers with equally spaced sticky monomers along the chain. ${ }^{49}$ These stickers can only form binary bonds, similar to those assumed in the sticky Rouse model. The mechanical properties of the system were studied under nonequilibrium condition by using creep and constant volume tension simulations. Simulation results on monomer diffusion, nonequilibrium chemical dynamics and nonlinear mechanical properties were understood in terms of the crossover from diffusion-limited to kinetically limited sticky bond recombination and chain connectivity. Bedrov et al. performed standard molecular dynamics simulations of short telechelic polymer solutions where the attractive Lennard-Jones interactions among the end groups lead to the formation of networks of interlinked micelles or end-group clusters. ${ }^{48}$ The stress relaxation in the system was elucidated as a two-step process, a first decay due to the translational motion of the end-groups inside their clusters and secondly by the rapid hopping diffusion of end-groups between neighboring clusters, which is followed by the terminal relaxation due to cluster disintegration. In the above-mentioned simulations, the spacers in between the stickers are still relatively short ( $6 \sim 15$ monomers), which limits the capacity of clearly identifying the contributions from the parent polymer dynamics and its interplay with the sticker hopping process, both of which play an important role in theoretical models of associating polymer networks.

In this work, we study the dynamics and rheology of supramolecular polymer networks using a model system consisting of unentangled telechelic polymers. The flexible polymer chains are represented by the Kremer-Grest bead-spring model. ${ }^{51}$ The end monomers of the chains or stickers can associate with each other to form reversible sticky bonds with controllable reaction kinetics. ${ }^{49}$ The functionality of the stickers is set to be $f=3$, meaning that each sticker can maximally associate with two other stickers. This is the minimum functionality required for percolated network formation. ${ }^{52}$ Telechelic chains with functionality of $f=2$ undergo head-to-tail associations, which have been studied in other theoretical and simulation works. ${ }^{50,53-55}$ The choice of $f=3$ could be traced to experimental supramolecular networks constructed by mixtures of associating ditopic (A2) and tritopic (B3) molecules. ${ }^{5,56,57}$ By making the sticky monomer 
association directional, this model can also be conveniently applied to study reversible networks formed by $\pi-\pi$ stacking ${ }^{11,12}$ or ureidopyrimidinone (UPy) stacking. ${ }^{58,59}$ More importantly, stickers with finite functionality can form clusters with well-defined size distribution in equilibrium state, which is essential for providing a clear microscopic picture of the relationship between the dynamics of the cross-links and the viscoelastic behavior in the reversible networks of interconnected clusters or micelles. Our simulations reveal that the dynamics and stress relaxation in such systems are dominated by the partner exchange process which is facilitated by the repeated dissociation and association of sticker clusters, rather than by the theoretically assumed single sticker hopping process. These findings can be applied to understand the dynamics in supramolecular polymer networks where the stickers aggregate into clusters or micelles and also allow for examination of assumptions made in the related theoretical models. ${ }^{27,31}$

The rest of this paper is organized as follows. In Section 2 we describe the polymer chain model and the hybrid MD/MC simulation method used in this study. Simulation results on the static, dynamic and rheological properties of supramolecular systems are presented and discussed in Section 3, together with some theoretical models developed for describing the dynamic behavior of reversible polymer networks. The conclusions are drawn in Section 4. Table 1 lists all the characteristic time scales used in this work.

\section{Models and Simulation Methods}

The telechelic polymers are represented by the flexible bead-spring model. ${ }^{51}$ Each chain consists of $N$ monomers with one monomer at each chain end defined as a sticker. The stickers are identical to normal monomers except that they are capable of reversibly associating with one another. With a fixed functionality of $f=3$, each sticker can maximally associate with two other stickers. It is prohibited for a given sticker to form both sticky bonds with the same other sticker. Otherwise the telechelic chains will tend to associate into long linear living polymers, instead of 3D transient networks that are the main interest of the current work. The sticker functionality value can easily be 
adjusted for modeling different polymer systems.

The monomer density in the systems is fixed at $\rho=0.85 / \sigma_{L J}^{3}$ where $\sigma_{L J}$ is the diameter of the monomers. This choice of $\rho$ has been widely used to simulate polymer melts. ${ }^{51}$ For flexible Kremer-Grest chains in the melt condition, the entanglement length is estimated to be in the range of $N_{e}=50 \sim 80$, depending on the analysis method used. ${ }^{60-62}$ Therefore we choose to study two polymer chain lengths, $N=25$ and 45 , in the unentangled regime, bearing in mind that there could occasionally be lockedin entanglements due to the reversible association of the end monomers. As will be seen in the stress modulus calculations, there is no significant contributions from such entanglements. By studying unentangled parent polymer chains we can focus on relating the dynamics of the cross-links to the dynamic and rheological behavior of the resulting transient networks.

All monomers in the system interact pairwise via the purely repulsive Lennard-Jones (LJ) potential

$$
U_{L J}(r)=4 \epsilon_{L J}\left[\left(\frac{\sigma_{L J}}{r}\right)^{12}-\left(\frac{\sigma_{L J}}{r}\right)^{6}-\left(\frac{\sigma_{L J}}{r_{c}}\right)^{12}+\left(\frac{\sigma_{L J}}{r_{c}}\right)^{6}\right]
$$

for $r \leq r_{c}$, where $r_{c}=2^{1 / 6} \sigma_{L J}$ is the cut-off radius and $U_{L J}(r)=0$ for $r>r_{c}$. The LJ interaction parameter is chosen to be $\epsilon_{L J}=1.0 k_{B} T$ where $k_{B}$ is the Boltzmann constant and $T$ is the absolute temperature. Each pair of adjacent beads in a chain interact via the finitely extensible non-elastic (FENE) potential

$$
U_{F E N E}(r)=-\frac{k R_{\max }^{2}}{2} \ln \left[1-\left(\frac{r}{R_{\max }}\right)^{2}\right]
$$

where $R_{\max }=1.5 \sigma_{L J}$ and $k=30 \epsilon_{L J} / \sigma_{L J}$. The system is coupled to the Langevin thermostat to maintain a constant temperature. The equations of motion of the monomers are solved numerically using the Verlet algorithm with a MD time step size $\delta t=$ $0.01 \tau_{L J}$ where the Lennard-Jones time $\tau_{L J}=\sqrt{m \sigma_{L J}^{2} / \epsilon_{L J}}$ and $m$ is the mass of the monomers. ${ }^{51,62,63}$ The simulations are carried out in the canonical (NVT) ensemble with periodic boundary conditions applied in all three directions. The stickers are allowed to associate across periodic boundaries. 
When two stickers form a reversible sticky bond, they interact via the potential ${ }^{49,54}$

$$
U_{s b}(r, \varepsilon)=U_{F E N E}(r)-U_{F E N E}\left(r_{0}\right)-\varepsilon
$$

where $r_{0} \approx 0.97 \sigma_{L J}$ is the equilibrium bond length at the minimum of the combined potential $U_{F E N E}(r)+U_{L J}(r)$. The energy offset $U_{F E N E}\left(r_{0}\right)+\varepsilon$ in eq.(3) is introduced to control the lifetime of the sticky bonds and consequently the fraction of associated stickers in the system. The sticky bonding energy $\varepsilon$ is independent of the separation between the two stickers and so does not alter their associating force. The formation and breaking of sticky bonds are controlled by the Metropolis Monte Carlo Algorithm ${ }^{64}$ where the energy change due to the formation of a new sticky bond is $\Delta E(r, \varepsilon)=$ $U_{s b}(r, \varepsilon)$ and the energy change to break an existing bond is $\Delta E(r, \varepsilon)=-U_{s b}(r, \varepsilon)$. If an $\mathrm{MC}$ move causes a reduction in the change of energy $\Delta E(r, \varepsilon) \leq 0$, it is always accepted. On the other hand, if $\Delta E(r, \varepsilon)>0$, a move is accepted with probability $\exp \left[-\Delta E(r, \varepsilon) / k_{B} T\right]$. The forming/breaking processes of the two sticky bonds for each sticker are treated independently. At each MC step pairs of stickers are chosen randomly. If the chosen pair are already bonded, an attempt is made to break the bond. Conversely, if the pair are not bonded, an attempt is made to create a sticky bond. Each pair is chosen on average once per MC step. The frequency $f_{M C}=\tau_{L J} / \tau_{M C}$ at which MC steps occur governs the reaction kinetics of the stickers. By increasing the MC time step size $\tau_{M C}$ the sticky bond relaxation is effectively changed from diffusion-limited to kinetically limited regime, which will consequently alter the dynamic behavior of the system, but not the thermodynamic or static properties. Most of the simulation data presented in this work were generated using $\tau_{M C}=0.01 \tau_{L J}$ (i.e., one MC step at each MD time step), with some extra runs using $\tau_{M C}=1.0 \tau_{L J}$ for comparison. As will be shown in the next section, the use of smaller $\tau_{M C}$ value leads to shorter terminal relaxation times of the systems and so enables us to obtain good statistical results with affordable computational efforts. It should be noted that the change of $\tau_{M C}$ values will not affect the qualitative results obtained in the equilibrium systems as studied here. Furthermore, Hoy and Fredrickson have shown that small MC time step sizes are 
needed to reduce systematic errors in calculating dynamic and mechanical properties of reversible associating polymer networks. ${ }^{49}$ Our hybrid MD/MC algorithm can be conveniently applied to study supramolecular polymer systems where the stickers have higher functionality $(f \geq 4)$ and so can associate into large clusters, such as via ionic or hydrophobic interactions. The slow dynamics of stickers in large clusters due to caging effects will dramatically slow down the dynamics and stress relaxation of the system. To simulate such systems will be computationally much more expensive than that required in the current work with $f=3$. One also needs to make sure that data analysis is carried out in equilibrium state. We will thus leave such systems for later study. We also noticed that for simulating systems with high sticker functionality the MC moves for the sticky bond formation and breakage can be alternatively treated by the method proposed by Daoulas et al. where the probability for a sticker to associate with one of its neighboring stickers (if not bonded) is determined by the Boltzmann weights of these neighbors. ${ }^{65}$

Each simulation system undergoes two stages of equilibration before any analysis takes place. At first the system is equilibrated as a polymer melt with all stickers treated as normal monomers along the chains. ${ }^{61,62}$ This stage lasts for a period of multiple Rouse times of the unentangled chains. As an example, the Rouse time for the flexible chains of length $N=25$ as used in our simulations is $\tau_{R} \approx 923 \tau_{L J}$. In the second stage the hybrid $\mathrm{MD} / \mathrm{MC}$ simulation are carried out with the sticker association mechanism switched on. This stage is considerably longer than the first one due to the much longer relaxation time of polymer chains in a supramolecular network than in a melt (typically increased by a factor of $5-10)$. Following the equilibration stages the static and dynamic properties of the reversible network are calculated on the fly over an equilibrium run of $10-100$ terminal relaxation times of the whole system.

The static, dynamic and rheological properties of the model systems are studied for a range of sticky bonding energy from $\varepsilon=0$, corresponding to regular polymer melt, up to $\varepsilon=12 k_{B} T$. As shown in Appendix A, the sol-gel transition of such systems takes place at $\varepsilon \approx 4.3 k_{B} T$, which is consistent with the critical $\varepsilon$ value found in simulation systems 
where sticky monomers interact with the same bonding potential as in eq. (3), but follow the binary bonding rule. ${ }^{49}$ The simulation box we used contains $N_{c h}=400$ polymer chains in case of polymerization $N=25$. For $N=45$ there are $N_{c h}=200$ chains. The finite size effects have been addressed in Appendix A and found to be insignificant in the current work. To improve the statistics, all simulation data on the reversible networks are averaged over at least 4 independent runs for each set of system parameters. Much larger ensemble averages are taken for the permanent networks generated by preventing the sticky bonds from dissociation, as will be seen in the next section.

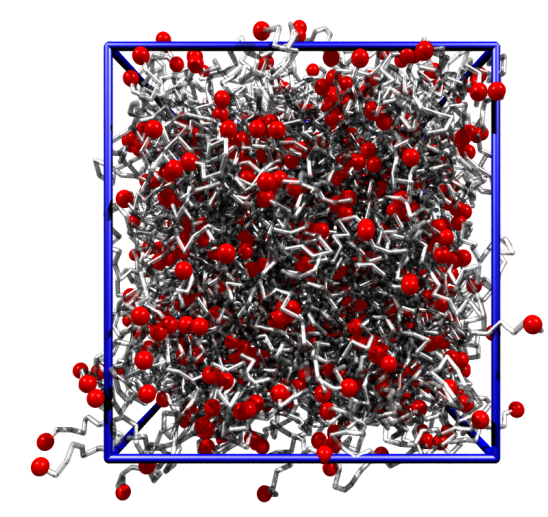

Figure 1: Snapshot of a transient network formed by associating telechelic chains of length $N=25$ and sticky bonding energy $\varepsilon=10 k_{B} T$. The red spheres represent the stickers at the chain ends.

\section{$3 \quad$ Results and Discussions}

\subsection{Static properties: reversible network analysis}

Fig. 1 presents a snapshot of the simulation system consisting of associating telechelic chains of length $N=25$ and sticky bonding energy $\varepsilon=10 k_{B} T$. It shows clearly that at high enough bonding energy, the stickers associate into clusters of different sizes which 
cross link the parent polymer chains into a transient network. The topological structures of the networks can thus be understood from the sticker cluster size distributions.

\subsubsection{Sticker cluster formation}

In our model systems each sticker can bond with up to two partners. This allows for three possible bonding states: open with no bonded partner, partially reacted with one bonded partner and fully reacted with two bonded partners. The average fraction of stickers in each state is calculated as a function of the bonding energy $\varepsilon$. The simulation results in Fig. 2 for the systems with chain length $N=25$ demonstrate that the fraction of open stickers decreases monotonically with the increase of $\varepsilon$, while the total fraction of (partially and fully) reacted monomers keeps on increasing and gradually saturates at high $\varepsilon$ values. The crossover of these two fraction curves occurs at $\varepsilon \approx 4.3 k_{B} T$ which is very close to the critical bonding energy for the sol-gel transition, see Appendix A. The fraction of fully reacted stickers becomes dominant when $\varepsilon>6 k_{B} T$. At high bonding energies $\varepsilon \geq 10 k_{B} T$, the majority of the stickers are fully reacted and the fraction of open stickers is down to less than $1 \%$. In the sticker hopping picture for binary bonding systems, ${ }^{22,29,30,34,49}$ if the fraction of open stickers is low, pairs of associated stickers usually break and recombine many times before finding other open stickers to associate with. This significantly slows down the dynamic relaxation behavior as recombination with previous partners leaves the network topology unchanged. In the transient networks we studied, the formation of larger sticker clusters can facilitate the partner exchange process as shown in Section 3.2.2.

Stickers with functionality $f=3$ (or above) can associate into clusters with various sizes. The cluster size distribution can be described by the probability for finding a sticker in a cluster of size $N_{c l u}$

$$
P\left(N_{c l u}\right)=\frac{n_{N_{c l u}} N_{c l u}}{2 N_{c h}}
$$

where $n_{N_{c l u}}$ is the average number of sticker clusters of size $N_{c l u}$ and $2 N_{c h}$ is the total number of stickers in the system. Simulation results on $P\left(N_{c l u}\right)$ for the two different 




Figure 2: Average fractions of stickers that are in open, partially reacted and fully reacted states as a function of sticky bonding energy $\varepsilon$. The chain length is $N=25$.

chain lengths at $\varepsilon=10 k_{B} T$ are given in Fig. 3(a). In agreement with the high reaction rate at this bonding energy (Fig. 2), the majority of the stickers aggregate into clusters with sizes $N_{c l u} \geq 3$. The distinct peak at $N_{c l u}=3$ corresponds to the smallest cluster size for which each sticker can be fully reacted and so gain $-\varepsilon$ in association energy. The cluster size distribution is determined by the competition between this energy gain and the entropic penalties due to the loss of sticker translational entropy. In solutions of associating polymers, the formation of sticker clusters or micelles can lead to elastic stretching of the polymer chains, which in turn affects the sizes of stable clusters. But this polymeric effect is negligible in melt conditions, because the average end-to-end distance of the polymer chains is nearly constant in systems with different $\varepsilon$ values.

Cluster formation of stickers in equilibrium state can be theoretically described in a similar way as micelle formation of amphiphilic molecules in dilute solutions. ${ }^{66}$ Equilibrium thermodynamics requires the mole fraction, $X_{N_{c l u}}$, of stickers associated into clusters of size $N_{c l u}$ to satisfy the condition

$$
\mu_{N_{c l u}}^{0}+\frac{k_{B} T}{N_{c l u}} \ln \left(X_{N_{c l u}} / N_{c l u}\right)=\text { const }
$$





Figure 3: (a) Probabilities for finding a sticker in a sticker cluster of size $N_{c l u}$ in the systems with sticky bonding energy $\varepsilon=10 k_{B} T$. The dashed curves illustrate the analytical results given by eq. (9). (b) Average sticker cluster size as a function of sticky bonding energy. 
where the chemical potential of a sticker inside a cluster is given by

$$
\mu_{N_{c l u}}^{0}=-\varepsilon+F^{p o l y}\left(N_{c l u}\right), \quad N_{c l u} \geq 3 .
$$

The second term on the right hand side of eq. (6) allows the inclusion of possible (positive) polymeric contributions to the free energy. Since the chemical potential has a minimum value of $\mu_{M_{c l u}}^{0}=-\varepsilon$ at the cluster size $M_{c l u}=3$, it is convenient to describe the mole fraction $X_{N_{c l u}}$ by ${ }^{66}$

$$
\frac{X_{N_{c l u}}}{N_{c l u}}=\left(\frac{X_{M_{c l u}}}{M_{c l u}} \exp \left[M_{c l u}\left(\mu_{M_{c l u}}^{0}-\mu_{N_{c l u}}^{0}\right) / k_{B} T\right]\right)^{N_{c l u} / M_{c l u}}, \quad N_{c l u} \geq M_{c l u}
$$

In our system of telechelic chains $X_{N_{c l u}}$ is related to the sticker density as

$$
\sum_{N_{c l u}=1}^{\infty} X_{N_{c l u}}=\frac{2}{N}
$$

Hence, the probability of finding a sticker in a cluster of size $N_{c l u}$ is related to $X_{N_{c l u}}$ by $P_{c l u}\left(N_{c l u}\right)=X_{N_{c l u}} N / 2$ where $\sum_{N_{c l u}} P_{c l u}\left(N_{c l u}\right)=1$. If we neglect all the polymeric effects by assuming $F^{\text {poly }}\left(N_{c l u}\right)=0$, eq.(7) can be simplified to

$$
P_{c l u}\left(N_{c l u} \geq 3\right)=N_{c l u}\left(\frac{2}{N}\right)^{N_{c l u} / 3-1}\left(\frac{P_{c l u}(3)}{3}\right)^{N_{c l u} / 3}
$$

where the only input parameter is $P\left(N_{c l u}=3\right)$ whose value can be found in simulations.

As shown in Fig. 3(a), the predictions of eq. (9) are in reasonably good agreement with the simulation data. The relatively faster decay of the theoretical curves can be attributed to the assumption of dilute solution of stickers made in developing eq. (7). Since the polymer chain lengths we studied are still relatively short, the small sticker clusters have a fairly high probability to meet each other and associate into larger clusters, leading to a slower decay of $P_{c l u}\left(N_{c l u}\right)$ at large $N_{c l u}$ values. When the chain length is increased from $N=25$ to 45 , the peak at $P_{c l u}\left(N_{c l u}=3\right)$ becomes higher and consequently the fraction of larger clusters gets smaller because of the reduced sticker density. The agreement between the theoretical prediction and simulation results also improves. 
Simulation results on the average sticker cluster sizes, defined as $N_{c l u}^{a v g}=\sum N_{c l u} P\left(N_{c l u}\right)$, are plotted in Fig. 3(b) as a function of $\varepsilon$. The value of $N_{c l u}^{a v g}$ first increases with the sticky bonding energy until $\varepsilon \approx 9 k_{B} T$ and then reaches a plateau, e. g., $N_{c l u}^{a v g} \approx 3.6$ for $N=25$. This is consistent with the results in Fig. 3(a) that at high $\varepsilon$ values more than $50 \%$ of stickers are in clusters of size 3 , because the chemical potential of stickers is minimized at $N_{c l u}=3$ for the functionality of $f=3$. The average sticker cluster size can be considered as the active functionality of junctions in a polymer network. ${ }^{67} \mathrm{As}$ will be shown in Section 3.2.2, the existence of larger clusters plays an essential role in determining the terminal relaxation times of the supramolecular systems.

\subsubsection{Elastically effective strands}

The mechanical strength of a polymer network is determined by the fraction of elastically effective strands. In unentangled networks each effective strand contributes to the rubbery modulus by an order of $k_{B} T{ }^{52}$ Apart from the reversible nature of cross-links, the transient networks formed by associating polymers have similar topological structures to chemically fixed networks and so posses elastically ineffective components, such as dangling chains and loops. This can already be seen in Fig. 2 from the nonzero fraction of open stickers even at the highest bonding energy studied. In addition, some of the partially reacted stickers are involved in the formation of sticker clusters of size two and consequently longer chains or network strands by the linear association of two or more parent polymer chains. This also reduces the modulus of the network.

We investigated the fraction of elastically effective strands in the transient networks using a method inspired by the primitive path analysis (PPA) of entangled polymers. ${ }^{60}$ This was done by randomly selecting instantaneous network configurations from the trajectories obtained in well-equilibrated hybrid $\mathrm{MD} / \mathrm{MC}$ simulations. The topological structures of these networks were fixed by preventing any existing sticky bonds from breaking in addition to stopping the creation of new sticky bonds. The excluded volume interactions among all monomers were then switched off to make the bonds contract and the system temperature was set to zero to remove thermal fluctuations. This results in 



Figure 4: (a) Fraction of elastically ineffective strands, $\phi_{\text {ineff }}$, in the supramolecular networks obtained by using different cutoffs to identify fully contracting chains in the PPA-type analysis. The inset presents the probability distributions of the chain end-toend distances in the fixed networks with both excluded volume interactions and thermal fluctuations switched off; (b) Direct MD simulation results on the stress relaxation of fixed polymer networks that are generated by fixing the topological structures of transient networks obtained from hybrid MD/MC simulations. All results are averaged over 100 statistically independent network configurations and the error bars show the standard deviation of the mean. The polymer chain length is $N=25$. 
the collapse of chains not contributing to the plateau modulus. The dangling chains shrink into single points, giving the chain end-to-end distance $R_{e e}=0$. The chain loops are somewhat different. Even though both ends of the loop belong to the same cluster or cross-link, they may still have a small separation $\left(R_{e e} \neq 0\right)$ because other stickers in the cluster are subject to tension along the shrunken network strands connected to them. For this reason, we need to introduce a cutoff distance for $R_{e e}$ to identify the ineffective strands.

In Fig. 4(a) we show the fraction of elastically ineffective chains, $\phi_{\text {ineff }}$, obtained from the PPA-type analysis of transient networks formed by telechelic polymers of length $N=25$. The $\phi_{\text {ineff }}$ values decrease with the use of smaller cutoffs and starts to converge after $R_{e e} \leq 0.75 \sigma_{L J}$. This is consistent with the probability distributions of the chain end-to-end distances given in the inset of Fig. 4(a) which show two distinct peaks at higher bonding energies with the minimum between the peaks occurring at $R_{e e} \approx$ $0.75 \sigma_{L J}$. The peak located at smaller $R_{e e}$ is indicative of chains that have collapsed, while the one at larger $R_{e e}$ represents the chains which contribute to the network elasticity. Therefore we can reasonably use this minimum location $\left(0.75 \sigma_{L J}\right)$ between these peaks as an approximation for the cutoff. It follows that there are about $5 \%$ ineffective strands in the networks formed at sticky bonding energies $\varepsilon \geq 10 k_{B} T$ when the average sticker cluster size nearly saturates, see Fig. 3. The strongly associated transient networks thus have high elastic efficiency. We note that unlike the PPA method our analysis algorithm does not preserve entanglements between the network strands. The cross-linking of unentangled polymer chains will unavoidably lock in a certain number of entanglements. How such entanglements contribute to the stress relaxation of the reversible networks should be investigated as a function of the parent chain length and sticky bonding energy, which will be left for further study.

To provide a reference for the plateau modulus of the reversible networks, we calculate the stress relaxation function, $G(t)$, of the fixed polymer networks used in Fig. 4(a) by performing standard MD simulations. The MD results on $G(t)$ are presented in Fig. 4(b) for network configurations taken from hybrid MD/MC simulations us- 
ing two different sticky bonding energies $\varepsilon=10 k_{B} T$ and $12 k_{B} T$. Each curve has been averaged over 100 statistically independent fixed network configurations. As expected the stress relaxation behaviors of the two sets of fixed networks agree with each other within error bars, confirming the similar topological structures of the reversible networks formed at high enough sticky bonding energies $\left(\varepsilon \geq 9 k_{B} T\right)$. The corresponding plateau modulus is $G_{N} \approx 0.028 k_{B} T / \sigma_{L J}^{3}$, which is close to the estimation of $G_{N}=\rho k_{B} T / N=0.034 k_{B} T / \sigma_{L J}^{3}$ for an ideal polymer network with monomer number density $\rho=0.85 \sigma_{L J}^{3}$ and strand length $N=25$.

\subsection{Dynamic and rheological properties}

A key difference of supramolecular polymer networks from polymer melts and permanent or chemical networks is the formation of reversible bonds. These introduce additional timescales into the systems, and consequently affects their dynamic and rheological behavior. We thus start with identifying the timescales characterizing the dynamics of reversible association of stickers and the underlying microscopic pictures, and then relate them to experimentally measurable properties, such as sticky monomer diffusion, stress and chain end-to-end vector relaxation functions.

\subsubsection{Timescales characterizing reversible association of stickers}

Sticky bonds are formed by physical association of pairs of stickers. Considering the dissociation of sticky bonds as a thermally activated process, their average lifetime, $\tau_{b}$, is predicted to depend exponentially on the bonding energy $\varepsilon^{34,49}$

$$
\tau_{b} \approx \tau_{M C} \exp \left(\varepsilon / k_{B} T\right)
$$

where the MC step size $\tau_{M C}$ reflects the controllable reaction rates of the stickers in the hybrid MD/MC simulation model. Fig. 5 presents the simulation data on $\tau_{b}$ for two different chain lengths and $\tau_{M C}=0.01 \tau_{L J}$, which follow the expected exponential dependence on $\varepsilon$. When increasing $\tau_{M C}$ from $0.01 \tau_{L J}$ to $1.0 \tau_{L J}$, the $\tau_{b}$ value was found to increase by a factor of about 100 without altering any static properties of the systems 
(results not shown). The average sticky bond lifetimes in the systems with longer chains $(N=45)$ are slightly larger than those in the shorter chain systems $(N=25)$. This can be attributed to the higher probability of stickers to form stable clusters (of size $N_{c l u}=3$, see Fig. 3) in the former systems, which effectively prolongs their average association time.

In the systems with sufficiently high bonding energies $\left(\varepsilon \geq 6 k_{B} T\right)$ most of the stickers are associated into clusters as shown in Fig. 2. Following a bond breaking event, the open stickers will most likely recombine with their old partners due to the low density of available open reaction sites nearby. This breaking and reforming process needs to be repeated many times before a sticker finally combines with new partners without returning to the old ones. It is through such partner exchange events that the topological constraint imposed by a sticker on its parent polymer chain is partly released. Therefore an additional timescale much longer than $\tau_{b}$ is required for describing the dynamic properties of associated polymer systems. ${ }^{22,34,49,68}$ In systems where stickers only experience binary bonding, a renormalized bond lifetime, $\tau_{b}^{*}$, was conveniently defined as the average time from the first moment that a sticker is bonded with one particular partner up to the moment that a bond is formed with a new open partner. ${ }^{34}$ The situation becomes more complicated for systems consisting of stickers with higher functionality $(f \geq 3)$ where larger sticker clusters are formed.

We introduce two timescales for characterizing the dynamics of releasing the topological constraints imposed by associated stickers. The first one is the partner exchange time, $\tau_{p e}$, which is defined as the average time taken for a given sticker from first being bonded with two particular partners until forming bonds with two new partners, as sketched in Fig. 6(a). This definition can be considered as an extension of the renormalized bond lifetime concept from the binary association cases $(f=2)$ to the systems with sticker functionality $f=3$. For a partner exchange event to take place there is no requirement for the two sticky bonds of a given sticker to break at the same time. Instead it may take multiple sticky bond formation and breaking steps until both partners of the sticker are exchanged. In many supramolecular systems the stickers can associate 




Figure 5: Average sticky bond lifetime $\tau_{b}$, partner exchange time $\tau_{p e}$ and cluster exchange time $\tau_{c e}$ with respect to sticky bonding energy $\varepsilon$ for the systems with two different chain lengths and $\tau_{M C}=0.01 \tau_{L J}$.

with more than two partners and the sticker clusters also have a broad size distribution, e. g., see Fig. 3. A more general definition of the characteristic timescale could be the cluster exchange time, $\tau_{c e}$, which is the time taken for a given sticker from being initially associated into one cluster consisting of three or more stickers until associating into another sticker cluster of size $N_{c l u} \geq 3$ which shares no other member stickers in common with the original cluster. This process can also occur in multiple steps with the member stickers within a cluster changing over time until none but the given one matches the original. The definition of $\tau_{c e}$ can be easily understood from the hopping picture of a sticker from one sticker cluster or micellar core to another, ${ }^{27,31}$ although this is not the dominant chain relaxation mechanism in the systems we studied as shown below. We note that these timescales are better defined in the strongly associated supramolecular networks than in the systems with low bonding energies. The latter cases are anyhow of little interest, because no transient network is formed and so chain dynamics are only weakly altered by the presence of stickers.

Fig. 6 compares the probability distributions of the partner exchange and cluster 
exchange times for the system with chain length $N=25$ and bonding energy $\varepsilon=10 k_{B} T$. At short time scales the cluster exchange events show higher probabilities than the partner exchange events, because the calculation of $\tau_{c e}$ also counts the events that involve partially-reacted stickers. In other words, a cluster exchange event is considered to be accomplished even if a given sticker is connected to a new cluster by a single sticky bond, while for finishing the partner exchange process the sticker is required to form sticky bonds with two new partners. The two probability distributions agree with each other reasonably well at timescales close to and beyond their average values. This agreement is expected for the systems with $f=3$ where more than $50 \%$ of the sticker clusters are of size 3. The ensemble-averaged values of $\tau_{p e}$ and $\tau_{c e}$ are presented in Fig. 5 as a function of $\varepsilon$ for the two different chain lengths. The two definitions provide nearly identical results within error bars (of symbol size). For convenience we will only use the partner exchange time $\tau_{p e}$ to represent these timescales in the remaining sections. This is also related to the later discussion on the microscopic picture of partner exchange events. The simulation data on $\tau_{p e}$ and also $\tau_{c e}$ can be fitted to an exponential function of the form

$$
\tau_{p e, c e} \approx \tau_{M C} \exp \left(B \varepsilon / k_{B} T\right)
$$

where $B=1.36>1$ indicates that the partner exchange time grows with $\varepsilon$ faster than the single exponential function of $\tau_{b} \sim \exp \left(\varepsilon / k_{B} T\right)$. This is qualitatively consistent with the renormalized bond lifetime $\tau^{*} \sim \exp \left(7 \varepsilon / 6 k_{B} T\right)$ predicted by Stukalin et al. for binary association of stickers at the ends of dangling chains. ${ }^{34}$ For a given bonding energy, the values of $\tau_{p e}$ and $\tau_{c e}$ are up to two orders of magnitude larger than the average bond lifetime $\tau_{b}$, indicating that $\tau_{b}$ is not sufficient for describing the dynamics in the supramolecular networks. Our simulation results are thus very different from the theoretical assumption that the bond lifetime renormalization is negligible in systems with micellar core formation, although the sticker bonding energy we studied does not fall exactly into the relevant range of $N_{c l u}^{1 / 2}<\varepsilon / k_{B} T<N_{c l u}^{4 / 3} \cdot{ }^{31}$ 




Figure 6: (a) Schematic demonstration of a partner exchange event which has a characteristic time $\tau_{p e}$; (b) Probability distributions of the partner exchange time $\tau_{p e}$ and cluster exchange time $\tau_{c e}$ for supramolecular systems with chain length $N=25$ and bonding energy $\varepsilon=10 k_{B} T$. 


\subsubsection{Microscopic picture of sticker partner exchange}

According to the original sticker hopping picture, a sticker first dissociates from the initial sticker cluster or micellar core and then diffuses as an open sticker until meeting another cluster to associate with. Although the difference in the total bonding energy of the sticker is negligible between the initial and final states, it needs to overcome an energy barrier on the order of $(f-1) \varepsilon$ to break all the sticky bonds formed in the initial cluster. In equilibrium systems the probability for such hopping events to happen is exponentially low, and the corresponding time scale would be proportional to $\exp \left[(f-1) \varepsilon / k_{B} T\right]$. But the simulation results on $\tau_{p e}$ or $\tau_{c e}$ in Fig. 5 grow with the association energy $\varepsilon$ much slower than $\exp \left(2 \varepsilon / k_{B} T\right)$ for $f=3$. This implies the existence of other pathways that have much lower energy barriers to allow the stickers to move from one cluster to another.

The sticker clusters in a supramolecular network fluctuate in space just like junction points in a permanent polymer network. When two smaller sticker clusters are in close proximity there is a high likelihood for them to associate into a large cluster due to the frequent breaking and formation processes of the sticky bonds under thermal fluctuations. Since the large cluster is entropically unfavorable and so short-lived, it will break apart into two new clusters which may or may not be of the same size as the two original ones, but has a relatively high probability to contain different member stickers. It is through this association-dissociation process of sticker clusters that stickers change their partners. Figure 7(a) sketches such a process where two sticker clusters both of size $N_{c l u}=3$ associate into a larger one of size 6 which later breaks into two new clusters to complete a partner exchange event. The cluster association-dissociation pathway thus facilitates the changes in the transient network topology without requiring stickers to fully dissociate from the network. In Fig. 7(a) the total number of sticky bonds remains 6 throughout the process, with sticky bonds frequently breaking and recombining under thermal fluctuations.

Based on the microscopic picture in Fig. 7(a), we perform a detailed analysis of partner exchange events, and correspondingly network topological changes, by studying 
the variation of sticker cluster size from the perspective of a sticker. Firstly, we define the stable sticker clusters as those possessing a lifetime larger than the average bond lifetime, $\Delta t>\tau_{b}$. Then we look at the transitions through which a sticker initially attached to a stable cluster finally associates with another stable cluster. We require the new cluster to either be of a new size or contain different members from the original cluster. This allows for three possible cases: 1) two clusters combine to form a larger cluster; 2) a smaller cluster breaks off from a larger cluster; 3) a cluster exchanges members with another cluster, but remains the same size. The events where two clusters combine together and then separate back into the original ones are not counted, because they do not result in changes in transient network topology. From the number of transitions we can determine a right-stochastic matrix, $M_{i, j}$, which measures the probability that a sticker initially in a cluster of size $N_{c l u}=i$ (initial state or $i$ th row in the matrix) transfers into a final cluster of size $j$ (final state or $j$ th column of the matrix). The matrix is described as right-stochastic because we normalize each row such that $\sum_{j} M_{i, j}=1$. The matrix is illustrated in Fig. 7(b) where the color of a block represents the magnitude of the transition probability.

Fig. 7 (b) shows that for $N_{c l u} \leq 3$ the clusters usually attempt to grow in size, e. g., from a cluster of size 2 to that of size 5 with $M_{2,5}=0.383$ (red block). This is contrasted by a usual decrease in cluster size when $N_{c l u} \geq 4$, e. g., from a cluster of size 7 to clusters of sizes 3 and 4 with $M_{7,3}=0.281$ (orange block) and $M_{7,4}=0.259$ (yellow block), respectively. It is evident that the most probable pathway for sticker cluster size changes is the addition or subtraction of three stickers, as marked by the two solid lines in Fig. 7(b). This can be understood by the fact that a group of three associated stickers has the maximum possible translational entropy without compromising bonding energy, as discussed in Section 3.1.1. On the contrary, the probabilities in the first column of the transition matrix are very low, indicating that it's very unlikely for a single sticker to break off a cluster. This further confirms that the partner exchange events usually take place via the cluster association and dissociation processes, rather than by single sticker hopping. The presence of large sticker clusters thus facilitates polymer chain relaxation 




Figure 7: (a) Sketch of a partner exchange event via the sticker cluster associationdissociation process; (b) Right-stochastic matrix that measures the probability for a sticker initially in a cluster of size $i$ ( $i$ th row) to transfer into a final cluster of size $j$ ( $j$ th column) for the system with $\varepsilon=10 k_{B} T$ and $N=25$. The two solid lines indicate the high probabilities for the cluster sizes to change by \pm 3 stickers. 
through the partner exchange process. In Section 3.2.4 we investigate how imposing an upper cap on the sticker cluster size would affect the stress relaxation behavior of the transient networks. We note that a sticker may need to experience multiple cluster association-dissociation events in order to exchange all of its original partners. This can be seen in the example sketched in Fig. 7(a) where stickers 1 and 4 have successfully exchanged both of their partners, while other stickers have only exchanged half of their original partners and so need more cluster association-dissociation events to exchange those which remain.

\subsubsection{Mean square displacement of stickers}

The effect of varying bonding energy on the mean square displacements (MSD) of stickers, $g_{1}^{\text {sticker }}(t)=\left\langle\left(\boldsymbol{r}^{\text {sticker }}(t)-\boldsymbol{r}^{\text {sticker }}(0)\right)^{2}\right\rangle$ where $\boldsymbol{r}^{\text {sticker }}(t)$ is the coordinate of the sticker center of mass, is shown in Fig. 8(a). For comparison we also include the MSD data of chain end monomers in polymer melts $\left(\varepsilon=0 k_{B} T\right)$ and of stickers in fixed polymer networks whose configurations were taken from the simulations of supramolecular systems with $\varepsilon=12 k_{B} T$. Since the parent chain lengths we studied are well below the entanglement segment length $N_{e}$, the monomer mean square displacements in the melt system follow Rouse-like behavior. At intermediate time scales $\tau_{0}<t<\tau_{R}$ where $\tau_{0}=\tau_{R} / N^{2}$, the end monomers diffuse faster than the middle monomers due to lower time-dependent effective friction. The ratio between the MSDs of the end and middle monomers has been shown previously to increase from 1 in the ballistic regime to a

plateau value around 2 close to the Rouse time $\tau_{R} \cdot{ }^{62,69}$ Beyond $\tau_{R}$ all monomers move coherently into the free diffusion regime, $g_{1}(t) \sim t^{1}$.

In supramolecular systems, the association of stickers significantly slows down their diffusion behavior. The transition from the subdiffusive to diffusive regime is delayed beyond the partner or cluster exchange time $\tau_{p e(c e)}\left(>\tau_{R}\right)$. At time scales $t<\tau_{p e}$, the MSD of the stickers is governed by the cluster size distribution. Fig. 8(b) shows the mean square displacements of sticker clusters of different sizes, $g_{1}^{N_{c l u}}(t)$, for the system with $\varepsilon=10 k_{B} T$. The MSD of stickers in clusters of size $N_{c l u}=2$ is analogous to that 
of middle monomers in chains of length $2 N$. As expected the growth rates of the $g_{1}^{c l u}$ curves decrease with the increase of $N_{\text {clu }}$. For each given bonding energy, the $g_{1}^{\text {sticker }}$ data in Fig. 8(a) can be exactly calculated by taking a weighted average of the cluster MSD results by using the cluster size distribution $P_{c l u}\left(N_{c l u}\right)$,

$$
g_{1}^{\text {sticker }}(t)=\sum_{N_{c l u}=1}^{\infty} P_{c l u}\left(N_{c l u}\right) g_{1}^{N_{c l u}}(t)
$$

up to the lifetimes of the related clusters.

The growth rate of $g_{1}^{\text {sticker }}(t)$ decreases with the increase of $\varepsilon$ as a consequence of the increased average cluster size. When $\varepsilon \geq 9 k_{B} T$ the average cluster size converges, e. g., to $N_{\text {avg }} \approx 3.6$ in systems with chain length $N=25$. Correspondingly the sticker MSD curves obtained at these high $\varepsilon$ values follow a universal behavior analogous to that resulting from the thermal fluctuations of cross-links in fixed polymer networks (dotted-dashed line) up to the partner exchange time in each case. This indicates that below $\tau_{p e}$ the supramolecular systems behave like permanent networks. At larger time scales $t>\tau_{p e}$, the stickers are able to exchange their partners through the cluster association-dissociation processes and so gradually forget their topological constraints. The $g_{1}^{\text {sticker }}(t)$ curves slowly cross over into the diffusive regime. Fig. 8 also shows that for the bonding energies studied in this work $\left(\varepsilon \leq 12 k_{B} T\right)$, there is still no extended plateau regime in the diffusion curves due to the limited lifetimes of the clusters.

The diffusion coefficients $D$ of the stickers and equivalently of the entire chains in the free diffusion regime are plotted as a function of $\varepsilon$ in the inset of Fig. 8(a). The decrease of the chain diffusivity with increasing sticker association energy has also been observed in experimental measurements of tracer chain diffusion in supramolecular polymer networks with different strengths of chain cross-linking. ${ }^{70,71}$ Our simulation data on $D$ show an exponential decay with $\varepsilon$ at higher bonding energies. As will be seen below, this is consistent with the exponential dependence of the chain terminal relaxation time $\tau_{d}$ on $\varepsilon$. 



Figure 8: (a) Mean-square displacements of stickers in supramolecular systems with different sticky bonding energies $\varepsilon$. The black dotted-dashed curve shows results obtained from fixed polymer networks whose configurations were taken from simulations of supramolecular systems with $\varepsilon=12 k_{B} T$. For reference the Rouse time $\tau_{R}$ of the chains and the sticker partner exchange time $\tau_{p e}$ at $\varepsilon=12 k_{B} T$ are given by the two vertical dashed lines on the right. The inset presents the diffusion coefficients of the stickers in the free diffusion regime as a function of sticky bonding energy. (b) MSD of sticker clusters with different sizes $N_{c l u} \leq 6$ at $\varepsilon=10 k_{B} T$. The parent chain length is $N=25$ in all cases. 

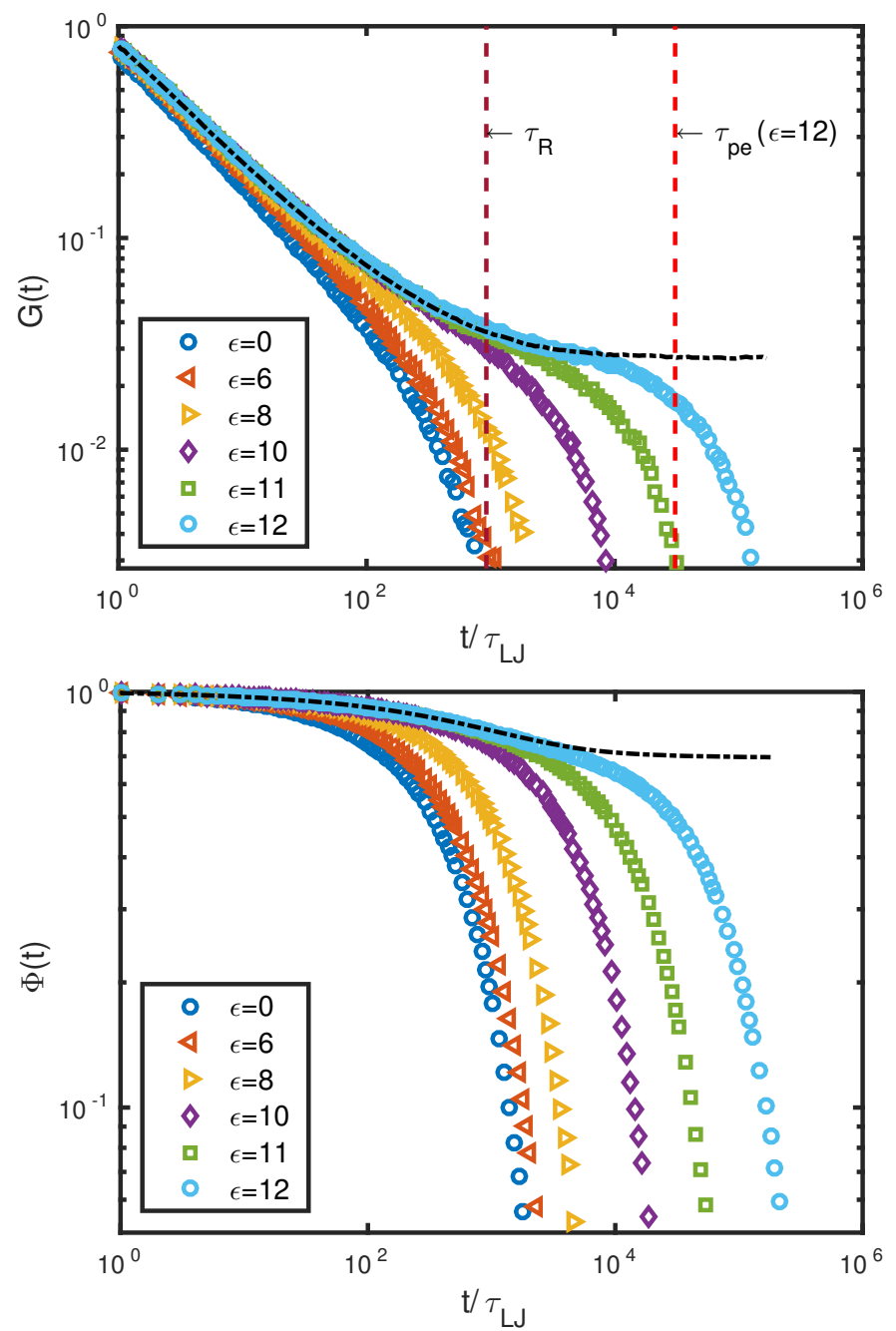

Figure 9: Stress relaxation (a) and chain end-to-end vector correlation function (b) in the systems with $N=25$ and various bonding energies $\varepsilon$. The dotted-dashed curves present the results obtained from fixed polymer networks whose configurations were taken from simulations of supramolecular systems with $\varepsilon=12 k_{B} T$. 


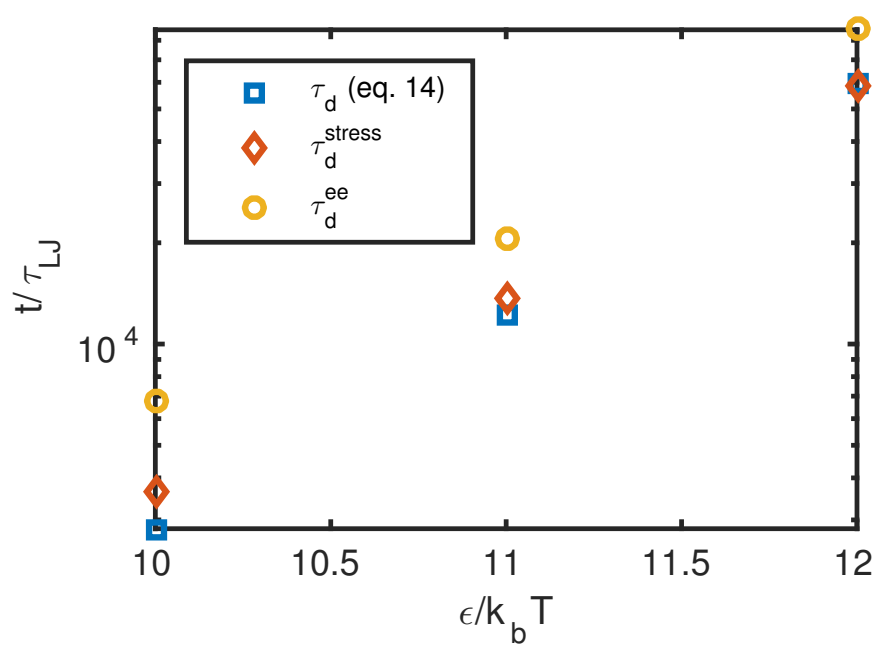

Figure 10: Simulation results on the terminal times of the stress relaxation, $\tau_{d}^{\text {stress }}$, and chain end-to-end vector correlation function, $\tau_{d}^{e e}$. The terminal relaxation times predicted by eq. (14) using simulation data on the sticker partner exchange time and hopping distance are also included.

\subsubsection{Stress and dielectric relaxation}

The reversible association of stickers also strongly affects the rheological behavior of the supramolecular systems. Fig. 9 presents the simulation results of the stress relaxation function, $G(t)$, and chain end-to-end vector correlation function or dielectric relaxation function, $\Phi(t)$, for the systems with $N=25$ and various bonding energies $\varepsilon$. Results obtained from polymer melts and fixed polymer networks are also included for comparison. All these time correlation functions were calculated on the fly using the multiple-tau correlator method to ensure good statistics. ${ }^{72}$

As the bonding energy $\varepsilon$ increases, the relaxation of the supramolecular systems demonstrates a gradual transition from the polymer melt-like behavior to fixed networklike behavior. In the systems with high enough $\varepsilon$ values where $\tau_{R}<\tau_{p e}$, three distinct relaxation regimes can be clearly identified in the $G(t)$ and $\Phi(t)$ curves: 1 ) initial Rouse regime at $\tau_{0}<t<<\tau_{R}$ where the relaxation curves follow universal Rouse-like behaviors, $G(t) \sim t^{-1 / 2}$ and $\left.\Phi(t) \sim t^{-1 / 2} ; 2\right)$ intermediate rubbery regime at $\tau_{R}<t<\tau_{p e}$ where the 
systems show rubber-like behavior due to the transient network formation; 3) terminal relaxation regime at $t>\tau_{p e}$ where the sticker partner exchange events lead to the stress and dielectric relaxation. Fig. 10 shows that at high sticky bonding energy the terminal times, $\tau_{d}^{\text {stress }}$ and $\tau_{d}^{e e}$, of the stress and chain end-to-end vector relaxation functions both grow exponentially with $\varepsilon$. There is roughly a factor of 2 difference between these two terminal times, but subject to rather poor statistics in $\tau_{d}^{\text {stress }}$ at high bonding energies. The analogy to the Rouse chain behavior $\left(\tau_{d}^{e e}=2 \tau_{d}^{\text {stress }}\right)^{73}$ implies that the release of topological constraints by partner exchange events takes place in a random-walk manner, and a theoretical model could be constructed based on this observation.

Earlier in Section 3.2.2 we described how the presence of large sticker clusters facilitates the partner exchange events. We now test this effect on the stress relaxation behavior directly by imposing an upper cap on the maximum size of the clusters, $N_{c l u}^{*}$, in simulations. Fig. 11 presents the stress relaxation functions for the systems with two different upper caps, namely $N_{c l u}^{*}=3$ and 4 respectively, together with that of the regular uncapped systems $\left(N_{c l u}^{*}=\infty\right)$. The $G(t)$ results show clearly that preventing the sticker clusters from growing in size leads to a much slower stress relaxation behavior in comparison with the regular supramolecular network we simulated, even though single sticker hopping events are allowed in both cases. Partner exchange events facilitated by the sticker cluster dissociation-association processes thus play a dominant role in controlling the dynamic and rheological behavior of supramolecular networks cross-linked by stickers cluster or micellar cores. In a theoretical work on the dynamics of telechelic ionomers, Leibler et al. have also pointed out that the stress relaxation should take place by exchanging pairs of charged chain ends to lower the free energy costs. ${ }^{74}$

\subsection{Theoretical models}

\subsubsection{Phantom chain hopping model}

In this section we introduce a simple theoretical model to describe the dynamic behavior of supramolecular polymer networks formed at high bonding energies $\left(\varepsilon \geq 9 k_{B} T\right)$ where nearly all stickers have associated into clusters. At time scales $t<\tau_{p e}$, the system 




Figure 11: Stress relaxation functions of supramolecular systems with and without a upper cap $N_{c l u}^{*}$ on sticker cluster size. The system parameters are $\varepsilon=10 k_{B} T$ and $N=25$.

behaves like a fixed polymer network and so can be described by the phantom network model where the sticker clusters act as cross-links or junctions. ${ }^{52}$ In a phantom network consisting of ideal-chain strands of length $N^{*}$ and cross-links of functionality $f^{*}$, each end monomer of a target network strand is considered to be effectively connected to an elastic non-fluctuating background via a virtual chain of length $N_{e f f}=N /\left(f^{*}-2\right)$. The other end, also called the anchor point, of the virtual chain is fixed in space. For mapping the phantom model to a supramolecular network formed by bead-spring chains with a broad distribution of sticker cluster sizes, we first choose a strand length $N^{*}$ and then match the time scales of the two systems by the ratio between the Rouse times of the phantom network strand and the parent polymer chains in the supramolecular system. Following that the virtual chain length $N_{e f f}$, or equivalently the effective crosslink functionality $f^{*}$, is determined by matching the mean square fluctuations of the end-to-end vectors of the network chains in the two different systems.

Unlike a permanent network, the end monomers or stickers of a polymer chain in a supramolecular network can change its topological connection to the network by moving 
from one sticker cluster or cross-link to another at time scales $t>\tau_{p e}$. The change of topological constraint on the target chain end via the partner exchange process can be represented by a hopping of the anchor point of the virtual chain in the phantom model. Fig. 12 sketches this phantom chain hopping model (PCHM) where a target Rouse chain consisting of $N^{*}$ beads is end-linked to two other Rouse chains each of $N_{\text {eff }}$ beads and anchored in space at the other end. The dynamics of the system is then controlled by the chain fluctuations and anchor point hopping.

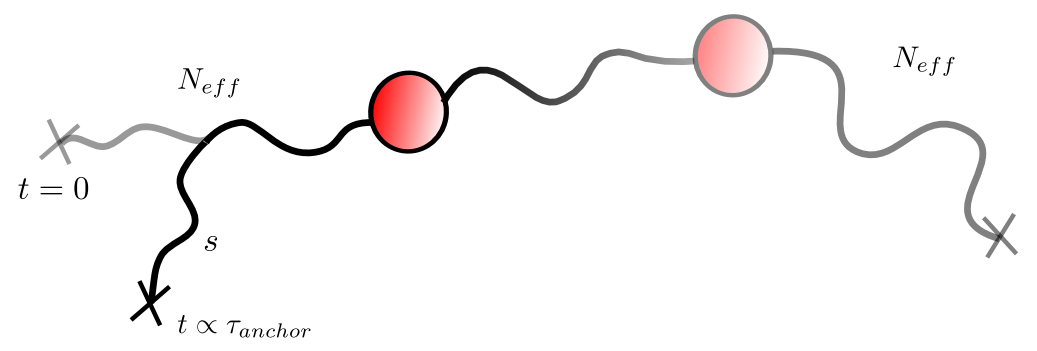

Figure 12: Sketch of the phantom hopping model. The red circles represent the stickers at the ends of the target chain.

The phantom chain hopping model can be solved numerically to provide dynamic relaxation functions of the target polymer. Apart from the two anchored beads, the dynamics of all other beads in the system are governed by the equations of motion ${ }^{73}$

$$
\zeta \frac{d \boldsymbol{R}_{i}}{d t}=-\frac{3 k_{B} T}{b^{2}}\left(\boldsymbol{R}_{i+1}-2 \boldsymbol{R}_{i}+\boldsymbol{R}_{i-1}\right)+\boldsymbol{f}_{i},
$$

where $\boldsymbol{R}_{i}$ is the Cartesian coordinate of bead $i, \zeta$ is the bead friction coefficient, $b$ is the average bond length and $\boldsymbol{f}_{i}$ is the Gaussian random force. Considering the broad distribution of the partner exchange times as shown in Fig. 6(b), each anchor point is assigned a lifetime $t_{\text {life }}^{*}$ randomly taken from a simple exponential distribution

$$
P\left(t_{\text {life }}^{*}\right)=P_{0} \exp \left(-t_{\text {life }}^{*} / \tau_{a n}\right)
$$

where $P_{0}$ is a normalization constant and $\tau_{a n}$ is the average anchor point lifetime whose value can be varied to reflect the dependence of the partner exchange time $\tau_{p e}$ on the bonding energy. For a given $\varepsilon$ value, we set $\tau_{a n}=\tau_{p e}\left(\tau_{R}^{*} / \tau_{R}\right)$ where $\tau_{R}^{*}$ and $\tau_{R}$ are 
the Rouse times of the target phantom network strand and the polymer chains in the supramolecular systems, respectively.

After $t_{\text {life }}^{*}$ the anchored bead performs a random hopping to a nearby position. The hopping process is carried out by eliminating a chain segment consisting of $s\left(\leq N_{\text {eff }}\right)$ beads from the anchored end of the virtual chain and then regenerating it by a $s$-step random walk of step size $b$, as sketched in Fig. 12. The position of the regenerated end monomer is taken as the new anchor point which is assigned a new lifetime from the distribution $P\left(t_{\text {life }}^{*}\right)$. The resulting anchor point hopping distance follows the Gaussian distribution of the end-to-end distance of an ideal chain with $2 s$ bonds and so has the mean value of $a_{a n}=(2 s)^{1 / 2} b$. In this algorithm, the impact of the abrupt hopping of the anchor point propagates to the related end monomer or sticker of the target chain through Rouse fluctuations of the virtual chain. Therefore the sticker can adapt to its new equilibrium position in the transient network smoothly, analogous to the partner exchange events in real supramolecular systems. The average hopping distance of the anchor point and correspondingly the number of hopping events needed for a target chain to fully relax can be tuned by changing the chain segment length $s$. The terminal relaxation of the target chain depends on both the mean anchor point lifetime $\tau_{a n}$ and the mean hopping distance $a_{a n}$. These essentially capture the effect of increasing the bonding energy and the sticker density as observed in the hybrid MD/MC simulations.

The system parameters of the phantom chain hopping model are set up as follows. For convenience we choose the number of beads in the target phantom chain same as that of the parent chains in the modeled supramolecular systems, i. e, $N^{*}=N$. To find the virtual chain length $N^{e f f}$ or the effective cross-link functionality $f^{*}$, we recall that in the phantom network model the mean square fluctuation of the end-to-end vector around its average value is given by $<\left(\boldsymbol{R}_{e e}-<\boldsymbol{R}_{e e}>\right)^{2}>=2 N^{*} b^{2} / f^{*}{ }^{75}$ Fig. 9 (b) shows that the end-to-end vector correlation function $\Phi(t)$ of the polymer chains with $N=25$ is relaxed by about $30 \%$ in the fixed supramolecular polymer networks. One can thus deduce the effective cross-link functionality by the relation of $2 / f^{*} \approx 0.3$ which gives $f^{*}=7$. This $f^{*}$ value is somewhat larger than the average sticker cluster 
size $N_{c l u}^{a v g} \approx 3.6$ found in the supramolecular networks. But as shown in Fig. 13 (a), the resulting virtual chain length of $N_{e f f}=5$ provides a very good prediction of the permanent phantom network model for the chain end-to-end vector correlation function in the fixed supramolecular networks. As mentioned above, the average lifetimes of the anchor points $\tau_{a n}$ are determined directly from the sticker partner exchange times $\tau_{p e}$ obtained in the hybrid MD/MC simulations at different bonding energies $\varepsilon$.

Fig. 13 presents the numerical results of the PCHM on the chain end-to-end vector correlation functions $\Phi(t)$ and the end-monomer or sticker mean square displacements $g_{1}^{\text {sticker }}(t)$ of the target chains, together with the MD/MC simulation data on supramolecular networks with $N=25$. At each $\varepsilon$ value the two sets of data show reasonably good agreement in both the Rouse and rubbery (plateau) relaxation regimes without requiring any extra tuning parameters. Further agreement in the terminal relaxation regime after $\tau_{a n}$ or $\tau_{p e}$ is achieved by choosing proper hopping distance $a_{a n}$ or the eliminated/regenerated chain segment length $s$. For example, a value of $s=4$ has been used for modeling the supramolecular systems with $N=25$ and $\varepsilon=10 k_{B} T$.

\subsubsection{Discrete model of sticker diffusion}

In supramolecular networks formed by associating telechelic chains, the topological constraints on the polymer chain ends are released in a step-by-step manner by sticker partner exchange events. The terminal relaxation time of the system can thus be estimated as the time taken for a sticker to diffuse a distance comparable to the size of its parent chain,

$$
\tau_{d} \approx \frac{N b^{2}}{a_{p e}^{2}} \tau_{p e}
$$

where $a_{p e}$ is the average distance that a sticker diffuses after one partner exchange event with the characteristic time $\tau_{p e}$. Eq.(14) takes a similar form as the free path (FP) model proposed by Marrucci et al. for equilibrium conditions, ${ }^{27}$ but the microscopic origins of the time and length scales of the discrete diffusion steps are very different from their model assumption.

In hybrid $\mathrm{MD} / \mathrm{MC}$ simulations, we define $a_{p e}$ as the separation between the mean 

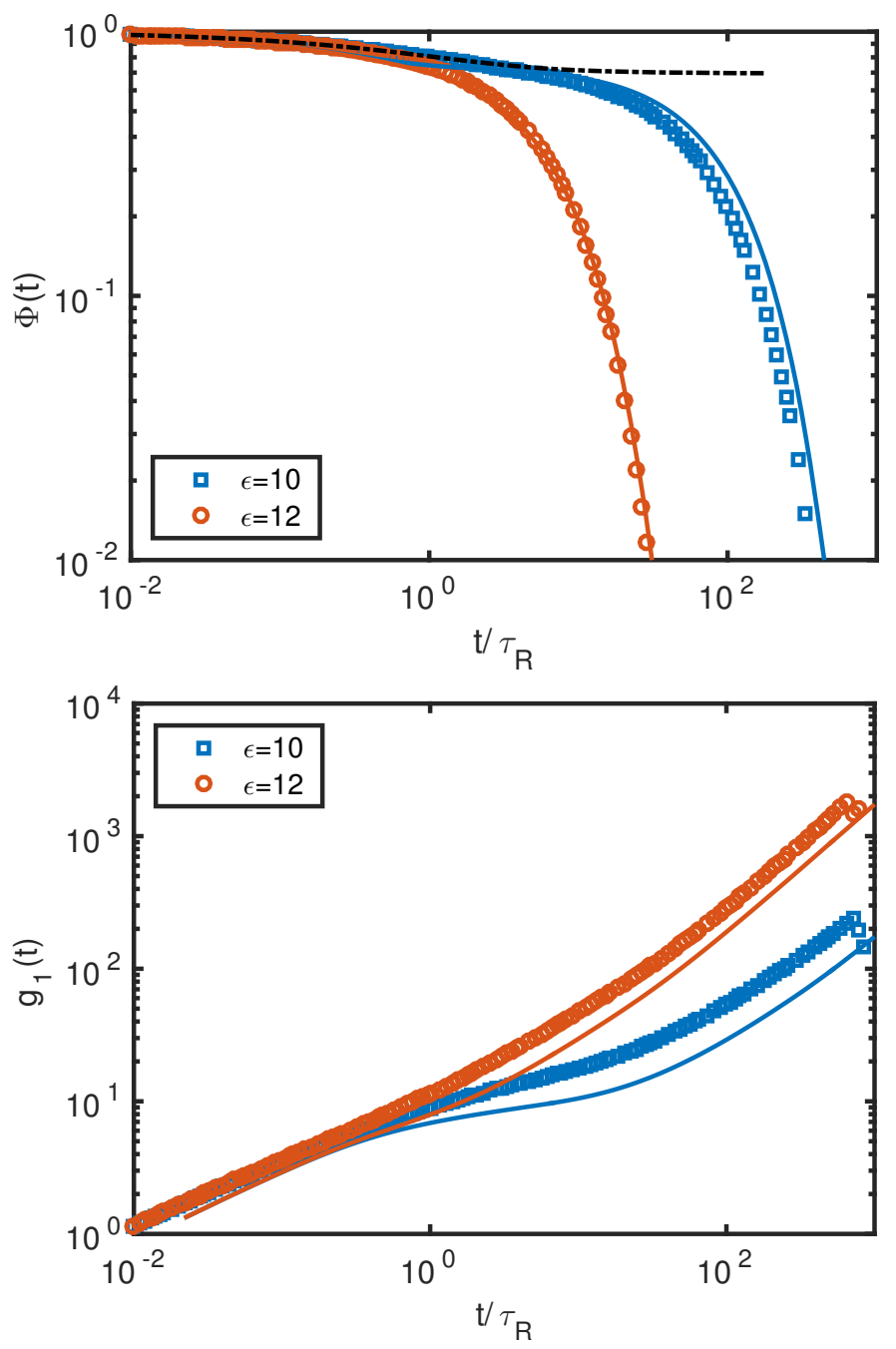

Figure 13: Simulation results of the phantom chain hopping model on the end-to-end vector correlation function $\Phi(t)$ (a) and the sticker mean square displacement (b) of the target chains. The symbols are the results obtained from hybrid MD/MC simulations of supramolecular networks with polymer chain length $N=25$ at various bonding energies $\varepsilon$. The simulation times in both the PCHM and supramolecular systems have been rescaled by the Rouse times of the corresponding polymer chains. The dotted-dashed line in (a) presents the $\Phi(t)$ data of the fixed polymer network same as in Fig. 9(b). 
positions of the initial and final sticker clusters that a sticker is associated with before and after a successful partner exchange event. The value of $a_{p e}$ is found to increase with the sticky bonding energy $\varepsilon$ even while the average size of clusters converges [Fig. 3(b)]. This implies that the sticker diffusion distance is determined by not only the average distance between sticker clusters, but also the lifetime of clusters. As the bonding energy increases, a cluster is able to explore a larger volume before the bond breakage permits its association with another cluster to facilitate sticker partner exchange. Fig. 10 compares the predictions of eq. (14) obtained by using the simulation values of $a_{p e}$ and $\tau_{p e}$ with the terminal times of the stress relaxation and chain end-to-end vector correlation functions of the supramolecular networks formed at high $\varepsilon$ values. They show qualitatively good agreement. But it should be noted that the statistics of the $a_{p e}$ and $\tau_{p e}$ values as well as the terminal times $\tau_{d}^{\text {stress }}$ and $\tau_{d}^{e e}$ are getting worse with increasing bonding energy, because the simulation runs can only last $15 \sim 200$ terminal relaxation times depending on $\varepsilon$.

We note that the sticker diffusion step size $a_{p e}$ used in eq. (14) is different from the anchor point hopping distance $a_{a n}$ defined in the phantom chain hopping model. But the random walk feature of the chain end diffusion can be well correlated to the Rouse-like relationship between the terminal times of the stress and chain end-to-end vector relaxation functions, namely $\tau_{d}^{e e} \approx 2 \tau_{d}^{\text {stress }}$. The PCH model has the advantage of being able to predict the entire relaxation functions over eq.(14) which only gives the terminal times.

\section{Conclusions}

Hybrid molecular dynamics/Monte Carlo simulations have been performed to study the static, dynamic and rheological properties of supramolecular systems consisting of unentangled telechelic chains with end sticky monomers. The choice of functionality $f=3$ allows each sticker to form reversible bonds with two other stickers, which is the minimum requirement for network formation. The sol-gel transition occurs at a critical 
sticky bonding energy of $\varepsilon \approx 4.3 k_{B} T$ when the fraction of reacted stickers overtakes that of the unreacted ones. At sufficiently high bonding energies $\left(\varepsilon \geq 10 k_{B} T\right)$, the majority of the stickers are fully reacted and less than one percent of stickers remain open. The distribution of the sticker cluster sizes can be well described by a theoretical model analogous to that used to predict micellar size distribution in dilute solutions of amphiphilic molecules. The proportion of elastically inefficient strands in the strongly associated supramolecular networks is found to be less than $5 \%$.

The dynamic and rheological behavior of the strongly associated supramolecular networks are shown to be dominated by partner exchange events in which the stickers exchange their associated partners, and so release the imposed topological constraints, through the disassociation-association processes of the sticker clusters. This is in contrast to the traditional picture of single sticker hopping where a sticker needs to dissociate from a cluster by breaking all existing sticky bonds, which is energetically unfavorable. Our study indicates that the system can relax without waiting for the chain ends to completely disassociate from the network. The presence of large sticker clusters can actually increase the chain relaxation rate. As a result preventing stickers from associating into larger clusters will significantly slow down stress relaxation.

Two characteristic time scales, namely the partner exchange time $\tau_{p e}$ and cluster exchange time $\tau_{c e}$, are introduced to measure the dynamics of supramolecular networks formed at high sticky bonding energies. These time scales are up to two orders of magnitude larger than the average sticky bond lifetime $\tau_{b}$. Three distinctive regimes can be identified in the stress and end-to-end vector relaxation functions, i. e., an initial Rouse regime at time scales $\tau_{0}<t<<\tau_{R}$, an intermediate rubbery or plateau regime at $\tau_{R}<t<\tau_{p e}$ and a terminal relaxation regime at $t>\tau_{p e}$. A phantom chain hopping model is developed based on the microscopic picture of sticker partner exchange process. Numerical predictions of this model on the sticker mean square displacements and chain end-to-end vector correlation functions are in reasonably good agreement with the hybrid MD/MC simulation results. Furthermore the terminal relaxation time of a supramolecular network can be estimated as the time taken for a sticker to diffuse a 
distance comparable to the size of its parent chain. The time and length scales of the discrete steps of the chain-end diffusion are determined by the sticker partner exchange events.

\section{Author Information}

Corresponding Author: * Email: zuowei.wang@reading.ac.uk

\section{Acknowledgments}

This paper is dedicated to our colleague and friend Professor Alexei E. Likhtman who sadly passed away during the publication of the manuscript. Alexei will be sorely missed by his family, friends, and colleagues.

We thank Evelyne van Ruymbeke and Patrick Ilg for helpful discussions. This work was supported by the Engineering and Physical Sciences Research Council (EPSRC) Grants No. EP/L020599/1 and EP/K017683/1.

\section{Appendix A: Sol-gel transition in supramolecular polymer systems}

In supramolecular polymer systems, the association of stickers leads to the formation of chain clusters of different sizes. For a system consisting of $N_{c h}$ telechelic chains with sticky end monomers of functionality $f$, the extent of reaction is measured by

$$
p=\frac{N_{\text {bond }}}{N_{c h}(f-1)}
$$

where $N_{\text {bond }}$ is the ensemble-averaged total number of sticky bonds formed in the system. The reaction extent $p$ increases with the increase of the sticky bonding energy $\varepsilon$. The sol-gel transition occurs when $p$ exceeds a critical threshold of $p_{c}$.

In order to determine if the system is percolated in a given direction we use the method of Koopman and Lowe ${ }^{76}$ which tests whether any group of associated chains is 


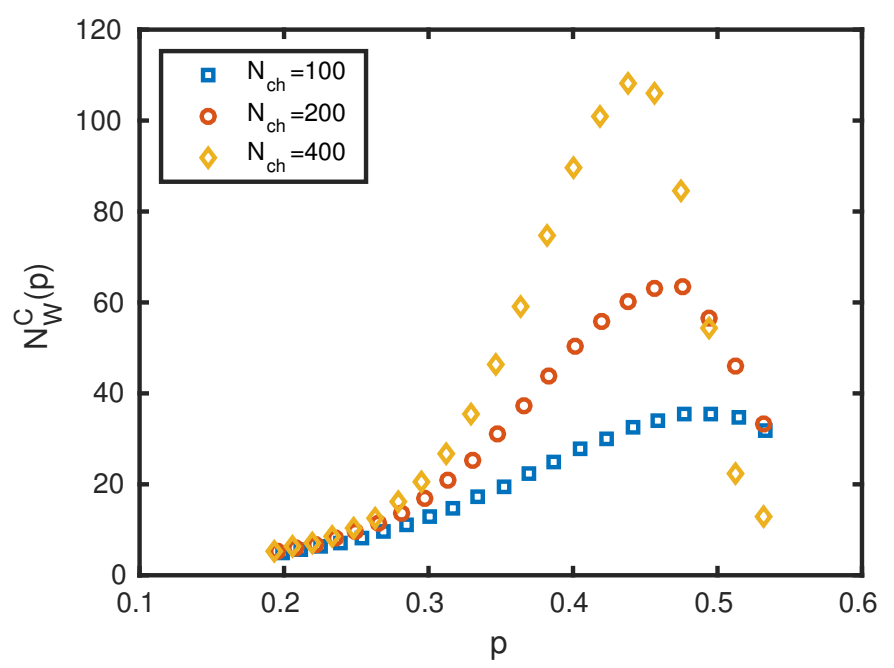

Figure 14: Weight-averaged chain cluster size as a function of the extent of reaction $p$ as obtained in supramolecular polymer systems with sticker functionality $f=3$. The simulations were performed using different box sizes and so different number of parent chains $N_{c h}$.

connected to its periodic image. We only require the system to be percolated in one direction. This analysis allows us to identify which chains make up the gel and which are part of the sol. The sol-gel transition can be characterized by the weight-averaged cluster size measured in the sol phase ${ }^{49,77}$

$$
N_{W}^{C}=\frac{\sum_{j=1}^{N_{c h}} j^{2} P_{s o l}(j)}{\sum_{j=1}^{N_{c h}} j P_{s o l}(j)} .
$$

where $P_{\text {sol }}(j)$ is the probability for a chain to be associated into a finite cluster consisting of $j$ chains. When $p$ approaches $p_{c}, N_{W}^{C}$ diverges in infinite system due to the formation of percolated network. However, since our simulations can only consider finite $N_{c h}$, a maximum in $N_{W}^{C}(p)$ is expected at the percolation transition.

Fig. 14 presents the simulation results on $N_{W}^{C}$ as a function of the extent of reaction as obtained in hybrid MD/MC simulations using different box sizes. The maximum of $N_{W}^{C}$ occurs at $p_{c} \approx 0.4$, which is in agreement with that found in systems of binary 
associations. ${ }^{49}$ This $p_{c}$ value corresponds to a sticker bonding energy $\varepsilon_{c} \approx 4.3 k_{B} T$. In Fig. 2 this bonding energy is approximately where the fraction of open stickers becomes less than the total fraction of (partially- and fully-)reacted stickers. Percolated transient networks are formed in the systems with $\varepsilon>\varepsilon_{c}$.

Table 1: List of symbols for the characteristic times used in this work.

\begin{tabular}{|l|l|}
\hline Timescale & Description \\
\hline$\tau_{L J}$ & Lennard-Jones time \\
\hline$\tau_{M C}$ & Monte Carlo step size \\
\hline$\tau_{R}$ & Rouse time of polymer chain \\
\hline$\tau_{d}$ & Terminal relaxation time of polymer chain \\
\hline$\tau_{d}^{e e}$ & Terminal relaxation time of polymer chain end-to-end vector \\
\hline$\tau_{d}^{\text {stress }}$ & Terminal time of stress relaxation \\
\hline$\tau_{b}$ & Average lifetime of sticky bonds \\
\hline$\tau_{b}^{*}$ & Renormalized lifetime of sticky bonds \\
\hline$\tau_{p e}$ & Partner exchange time of stickers \\
\hline$\tau_{c e}$ & Sticker cluster exchange time \\
\hline$\tau_{a n}$ & Average lifetime of anchor points in the phantom chain hopping model \\
\hline
\end{tabular}




\section{References}

[1] de Greef, T. F. A.; Meijer, E. W. Materials Science - Supramolecular Polymers. Nature 2008, 453, 171-173

[2] Seiffert, S.; Sprakel, J. Physical chemistry of supramolecular Polymer networks. Chem. Soc. Rev. 2012, 41, 909-930

[3] Serpe, M. J.; Craig, S. L. Physical organic chemistry of supramolecular Polymers. Langmuir 2007, 23, 1626-1634

[4] Syrett, J. A.; Becer, C. R.; Haddleton, D. M. Self-healing and self-mendable Polymers. Polym. Chem. 2010, 1, 978-987

[5] Cordier, P.; Tournilhac, F.; Soulié-Ziakovic, C.; Leibler, L. Self-healing and thermoreversible rubber from supramolecular assembly. Nature 2008, 451, 977-980

[6] Sijbesma, R.; Beijer, F.; Brunsveld, L.; Folmer, B.; Hirschberg, J.; Lange, R.; Lowe, J.; Meijer, E. Reversible Polymers formed from self-complementary monomers using quadruple hydrogen bonding. Science 1997, 278, 1601-1604

[7] Wietor, J.-L.; van Beek, D. J. M.; Peters, G. W.; Mendes, E.; Sijbesmat, R. P. Effects of Branching and Crystallization on Rheology of Polycaprolactone Supramolecular Polymers with Ureidopyrimidinone End Groups. Macromolecules 2011, 44, $1211-1219$

[8] Lewis, C. L.; Stewart, K.; Anthamatten, M. The Influence of Hydrogen Bonding Side-Groups on Viscoelastic Behavior of Linear and Network Polymers. Macromolecules 2014, 47, 729-740

[9] Li, J.; Viveros, J. A.; Wrue, M. H.; Anthamatten, M. Shape-memory effects in Polymer networks containing reversibly associating side-groups. Advanced Materials 2007, 19, 2851+ 
[10] Feldman, K. E.; Kade, M. J.; Meijer, E. W.; Hawker, C. J.; Kramer, E. J. Model Transient Networks from Strongly Hydrogen-Bonded Polymers. Macromolecules 2009, 42, 9072-9081

[11] Burattini, S.; Colquhoun, H. M.; Fox, J. D.; Friedmann, D.; Greenland, B. W.; Harris, P. J. F.; Hayes, W.; Mackay, M. E.; Rowan, S. J. A self-repairing, supramolecular Polymer system: healability as a consequence of donor-acceptor pi-pi stacking interactions. Chemical Communications 2009, 6717-6719

[12] Burattini, S.; Greenland, B. W.; Merino, D. H.; Weng, W.; Seppala, J.; Colquhoun, H. M.; Hayes, W.; Mackay, M. E.; Hamley, I. W.; Rowan, S. J. A Healable Supramolecular Polymer Blend Based on Aromatic $\pi-\pi$ Stacking and Hydrogen-Bonding Interactions. Journal of the American Chemical Society 2010, 132, 12051-12058

[13] Yount, W.; Loveless, D.; Craig, S. Strong means slow: Dynamic contributions to the bulk mechanical properties of supramolecular networks. Angewandte ChemieInternational Edition 2005, 44, 2746-2748

[14] Yount, W.; Loveless, D.; Craig, S. Small-molecule dynamics and mechanisms underlying the macroscopic mechanical properties of coordinatively cross-linked Polymer networks. Journal of the American Chemical Society 2005, 127, 14488-14496

[15] Loveless, D.; Jeon, S.; Craig, S. Rational control of viscoelastic properties in multicomponent associative Polymer networks. Macromolecules 2005, 38, 10171-10177

[16] Eisenberg, A.; Hird, B.; Moore, R. B. A new multiplet-cluster model for the morphology of random ionomers. Macromolecules 1990, 23, 4098-4107

[17] Chen, Q.; Tudryn, G. J.; Colby, R. H. Ionomer dynamics and the sticky Rouse model. Journal of Rheology 2013, 57, 1441-1462

[18] Chen, Q.; Masser, H.; Shiau, H.-S.; Liang, S.; Runt, J.; Painter, P. C.; Colby, R. H. 
Linear Viscoelasticity and Fourier Transform Infrared Spectroscopy of PolyetherEster-Sulfonate CoPolymer Ionomers. Macromolecules 2014, 47, 3635-3644

[19] Varley, R. J.; van der Zwaag, S. Towards an understanding of thermally activated self-healing of an ionomer system during ballistic penetration. Acta Materialia 2008, 56, 5737-5750

[20] Yamauchi, K.; Lizotte, J.; Long, T. Thermoreversible poly(alkyl acrylates) consisting of self-complementary multiple hydrogen bonding. Macromolecules 2003, 36, $1083-1088$

[21] Ahmadi, M.; Hawke, L. G. D.; Goldansaz, H.; van Ruymbeke, E. Dynamics of Entangled Linear Supramolecular Chains with Sticky Side Groups: Influence of Hindered Fluctuations. Macromolecules 2015, 48, 7300-7310

[22] Rubinstein, M.; Semenov, A. N. Dynamics of entangled solutions of associating Polymers. Macromolecules 2001, 34, 1058-1068

[23] Williams, C. E.; Russell, T. P.; Jerome, R.; Horrion, J. Ionic aggregation in model ionomers. Macromolecules 1986, 19, 2877-2884

[24] Muller, M.; Dardin, A.; Seidel, U.; Balsamo, V.; Ivan, B.; Spiess, H.; Stadler, R. Junction dynamics in telechelic hydrogen bonded polyisobutylene networks. Macromolecules 1996, 29, 2577-2583

[25] Rubinstein, M.; Dobrynin, A. Solutions of associative Polymers. Trends In Polymer Science 1997, 5, 181-186

[26] Baxandall, L. G. Dynamics of reversibly cross-linked chains. Macromolecules 1989, 22, 1982-1988

[27] Marrucci, G.; Bhargava, S.; Cooper, S. L. Models of shear-thickening behavior in physically cross-linked networks. Macromolecules 1993, 26, 6483-6488 
[28] Semenov, A. N.; Rubinstein, M. Thermoreversible Gelations in Solutions of Associative Polymers. Macromolecules 1998, 31, 1373-1385

[29] Rubinstein, M.; Semenov, A. N. Thermoreversible Gelations in Solutions of Associative Polymers. 2. Linear Dynamics. Macromolecules 1998, 31, 1386-1397

[30] Leibler, L.; Rubinstein, M.; Colby, R. H. Dynamics of reversible networks. Macromolecules 1991, 24, 4701-4707

[31] Semenov, A. N.; Rubinstein, M. Dynamics of entangled associating Polymers with large aggregates. Macromolecules 2002, 35, 4821-4837

[32] Hawke, L. G. D.; Ahmadi, M.; Goldansaz, H.; van Ruymbeke, E. Viscoelastic properties of linear associating poly(n-butyl acrylate) chains. Journal of Rheology 2016, $60,297-310$

[33] Indei, T.; Schieber, J. D.; Takimoto, J. Effects of fluctuations of cross-linking points on viscoelastic properties of associating polymer networks. Rheologica Acta 2012, 51, 1021-1039

[34] Stukalin, E. B.; Cai, L.-H.; Kumar, N. A.; Leibler, L.; Rubinstein, M. Self-Healing of Unentangled Polymer Networks with Reversible Bonds. Macromolecules 2013, $46,7525-7541$

[35] Colby, R.; Zheng, X.; Rafailovich, M.; Sokolov, J.; Peiffer, D.; Schwarz, S.; Strzhemechny, Y.; Nguyen, D. Dynamics of lightly sulfonated polystyrene ionomers. Physical Review Letters 1998, 81, 3876-3879

[36] Tierney, N.; Register, R. Ion hopping in ethylene-methacrylic acid ionomer melts as probed by rheometry and cation diffusion measurements. Macromolecules $\mathbf{2 0 0 2}$, 35, 2358-2364

[37] Groot, R. D.; Agterof, W. G. M. Monte Carlo study of associative polymer networks. 1. Equation of state. Journal of Chemical Physics 1994, 100, 1649-1656 
[38] Liu, Y.; Pandey, R. Sol-gel phase transitions in thermoreversible gels: Onset of gelation and melting. Journal of Chemical Physics 1996, 105, 825-836

[39] Kumar, Sanat K. and Douglas, Jack F., Gelation in Physically Associating Polymer Solutions. Phys. Rev. Lett. 2001, 87, 188301

[40] Manassero, C.; Raos, G.; Allegra, G. Structure of model telechelic Polymer melts by computer simulation. Journal of Macromolecular Science-Physics 2005, B44, $855-871$

[41] Guo, L.; Luijten, E. Reversible gel formation of triblock coPolymers studied by molecular dynamics simulation. Journal of Polymer Science Part B-Polymer Physics 2005, 43, 959-969

[42] Loverde, S.; Ermoshkin, A.; De La Cruz, M. Thermodynamics of reversibly associating ideal chains. Journal of Polymer Science PART B-Polymer Physics 2005, 43, 796-804

[43] Baljon, A. R. C.; Flynn, D.; Krawzsenek, D. Numerical study of the gel transition in reversible associating Polymers. Journal of Chemical Physics 2007, 126

[44] Goswami, M.; Kumar, S. K.; Bhattacharya, A.; Douglas, J. F. Computer simulations of lonomer self-assembly and dynamics. Macromolecules 2007, 40, 4113-4118

[45] Billen, J.; Wilson, M.; Rabinovitch, A.; Baljon, A. R. C. Topological changes at the gel transition of a reversible Polymeric network. Europhys. Lett. 2009, 87

[46] Wilson, M.; Rabinovitch, A.; Baljon, A. R. C. Aggregation kinetics of a simulated telechelic Polymer. Phys. Rev. E 2011, 84

[47] Groot, R. D.; Agterof, W. G. M. Monte Carlo study of associative polymer networks. 2. Rheologic aspects. Journal of Chemical Physics 1994, 100, 1657-1664

[48] Bedrov, D.; Smith, G.; Douglas, J. Influence of self-assembly on dynamical and viscoelastic properties of telechelic Polymer solutions. Europhys. Lett. 2002, 59, $384-390$ 
[49] Hoy, R. S.; Fredrickson, G. H. Thermoreversible associating Polymer networks. I. Interplay of thermodynamics, Chemical kinetics, and Polymer Physics. J. Chem. Phys. 2009, 131, 224902

[50] Li, Z.; Djohari, H.; Dormidontova, E. E. Molecular dynamics simulations of supramolecular polymer rheology. The Journal of Chemical Physics 2010, 133, 184904

[51] Kremer, K.; Grest, G. S. Dynamics of entangled linear polymer melts - a moleculardynamics simulation. J. Chem. Phys. 1990, 92, 5057-5086

[52] Rubinstein, M.; Colby, R. H. Polymer Physics, 1st ed.; Oxford University Press: Oxford New York, 2003

[53] Cates, M. E. Reptation of living polymers: dynamics of entangled polymers in the presence of reversible chain-scission reactions. Macromolecules 1987, 20, 2289-2296

[54] Huang, C. C.; Xu, H.; Ryckaert, J. P. Kinetics and dynamic properties of equilibrium polymers. J. Chem. Phys. 2006, 125, 094901

[55] Watanabe, H.; Matsumiya, Y.; Masubuchi, Y.; Urakawa, O.; Inoue, T. Viscoelastic Relaxation of Rouse Chains undergoing Head-to-Head Association and Dissociation: Motional Coupling through Chemical Equilibrium. Macromolecules 2015, 48, 30143030

[56] Deng, G.; Tang, C.; Li, F.; Jiang, H.; Chen, Y. Covalent Cross-Linked Polymer Gels with Reversible Sol-Gel Transition and Self-Healing Properties. Macromolecules 2010, 43, 1191-1194

[57] Liu, F.; Li, F.; Deng, G.; Chen, Y.; Zhang, B.; Zhang, J.; Liu, C.-Y. Rheological Images of Dynamic Covalent Polymer Networks and Mechanisms behind Mechanical and Self-Healing Properties. Macromolecules 2012, 45, 1636-1645

[58] van Beek, D. J. M.; Spiering, A. J. H.; Peters, G. W. M.; te Nijenhuis, K.; Sijbesma, R. P. Unidirectional dimerization and stacking of ureidopyrimidinone end 
groups in polycaprolactone supramolecular Polymers. Macromolecules 2007, 40, $8464-8475$

[59] Nieuwenhuizen, M.; deGreef, T.; vanderBruggen, R.; Paulusse, J.; Appel, W.; Smulders, M.; Sijbesma, R.; Meijer, E. Self-Assembly of Ureido-Pyrimidinone Dimers into One-Dimensional Stacks by Lateral Hydrogen Bonding. Chemistry A European Journal 2010, 16, 1601-1612

[60] Everaers, R.; Sukumaran, S. K.; Grest, G. S.; Svaneborg, C.; Kremer, A. S. A. Rheology and microscopic topology of entangled Polymeric liquids. Science 2004, 303, 823-826

[61] Likhtman, A. E.; Sukumaran, S. K.; Ramirez, J. Linear Viscoelasticity from Molecular Dynamics Simulation of Entangled Polymers. Macromolecules 2007, 40, 67486757

[62] Wang, Z.; Likhtman, A. E.; Larson, R. G. Segmental Dynamics in Entangled Linear Polymer Melts. Macromolecules 2012, 45, 3557-3570

[63] Allen, M. P.; Tildesley, D. J. Computer Simulation of Liquids; Clarendon Press: Oxford, 1987

[64] Frenkel, D.; Smit, B. Understanding Molecular Simulation: From Algorithms to Applications, 2nd ed.; Computational Science Series; Academic Press: San Diego, 2002

[65] Daoulas, K. C.; Cavallo, A.; Shenhar, R.; Muller, M. Phase behaviour of quasi-block copolymers: A DFT-based Monte-Carlo study. Soft Matter 2009, 5, 4499-4509

[66] Israelachvili, J. N.; Mitchell, D. J.; Ninham, B. W. Theory of self-assembly of hydrocarbon amphiphiles into micelles and bilayers. J. Chem. Soc., Faraday Trans. 2 1976, 72, 1525-1568

[67] Lang, M. Monomer Fluctuations and the Distribution of Residual Bond Orientations in Polymer Networks. Macromolecules 2013, 46, 9782-9797 
[68] Nyrkova, I. A.; Semenov, A. N. Correlation effects in dynamics of living Polymers. Europhys. Lett. 2007, 79, 66007

[69] Kreer, T.; Baschnagel, J.; Muller, M.; Binder, K. Monte Carlo simulation of long chain Polymer melts: Crossover from Rouse to reptation dynamics. Macromolecules 2001, 34, 1105-1117

[70] Hackelbusch, S.; Rossow, T.; van Assenbergh, P.; Seiffert, S. Chain Dynamics in Supramolecular Polymer Networks. Macromolecules 2013, 46, 6273-6286

[71] Rossow, T.; Habicht, A.; Seiffert, S. Relaxation and Dynamics in Transient Polymer Model Networks. Macromolecules 2014, 47, 6473-6482

[72] Ramirez, J.; Sukumaran, S. K.; Vorselaars, B.; Likhtman, A. E. Efficient on the fly calculation of time correlation functions in computer simulations. J. Chem. Phys. 2010, 133, 154103

[73] Doi, M.; Edwards, S. F. The theory of Polymer dynamics, 1st ed.; Claredon Press: Oxford, 1988

[74] Leibler, L.; Rubinstein, M.; Colby, R. H. Dynamics of telechelic ionomers. Can Polymers diffuse large distances without relaxing stress. J. Phys. II Frances 1993, 3, 1581-1590

[75] Rubinstein, M.; Panyukov, S. Elasticity of Polymer Networks. Macromolecules 2002, 35, 6670-6686

[76] Koopman, E.; Lowe, C. An algorithm for detecting percolating structures in periodic systems Application to polymer networks. Journal of Computational Physics 2014, $274,758-769$

[77] Pandey, R. B.; Landau, D. P. Directed growth in kinetic gelation. Journal of Physics A: Mathematical and General 1985, 18, L399 


\section{for Table of Contents use only}

Dynamics in Supramolecular Polymer Networks Formed by Associating Telechelic Chains Dipesh Amin, Alexei E. Likhtman and Zuowei Wang

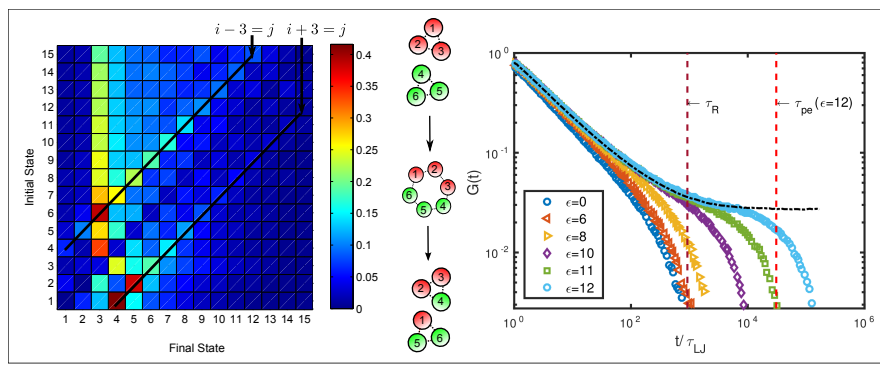

